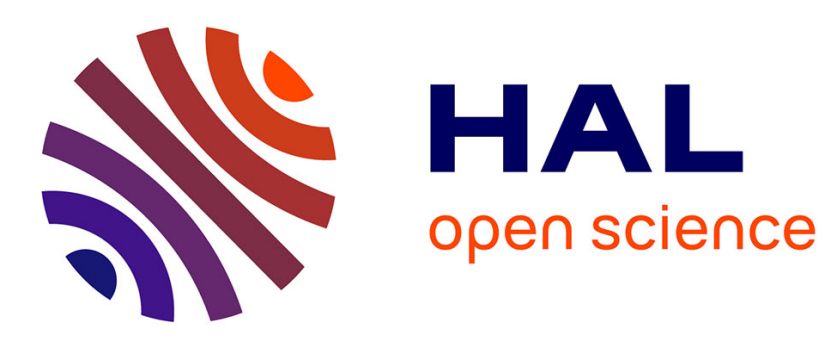

\title{
Note: Void effects on eddy current distortion in two-phase liquid metal
}

Mithlesh Kumar, Philippe Tordjeman, Wladimir Bergez, Matthieu Cavaro

\section{To cite this version:}

Mithlesh Kumar, Philippe Tordjeman, Wladimir Bergez, Matthieu Cavaro. Note: Void effects on eddy current distortion in two-phase liquid metal. Review of Scientific Instruments, 2015, 86 (10), pp.106104. 10.1063/1.4932990 . hal-01284031

\section{HAL Id: hal-01284031 \\ https://hal.science/hal-01284031}

Submitted on 7 Mar 2016

HAL is a multi-disciplinary open access archive for the deposit and dissemination of scientific research documents, whether they are published or not. The documents may come from teaching and research institutions in France or abroad, or from public or private research centers.
L'archive ouverte pluridisciplinaire HAL, est destinée au dépôt et à la diffusion de documents scientifiques de niveau recherche, publiés ou non, émanant des établissements d'enseignement et de recherche français ou étrangers, des laboratoires publics ou privés. 


\section{OATAO \\ Open Archive Toulouse Archive Ouverte}

\section{Open Archive TOULOUSE Archive Ouverte (OATAO)}

OATAO is an open access repository that collects the work of Toulouse researchers and makes it freely available over the web where possible.

This is an author-deposited version published in : http://oatao.univ-toulouse.fr/ Eprints ID : 15516

To link to this article : DOI:10.1063/1.4932990

URL : http://dx.doi.org/10.1063/1.4932990

To cite this version : Kumar, Mithlesh and Tordjeman, Philippe and Bergez, Wladimir and Cavaro, Matthieu Note: Void effects on eddy current distortion in two-phase liquid metal. (2015) Review of Scientific Instruments, vol. 86 ( $\left.\mathrm{n}^{\circ} 10\right)$. pp. 106104-106104. ISSN 0034-6748

Any correspondance concerning this service should be sent to the repository administrator: staff-oatao@listes-diff.inp-toulouse.fr 


\title{
Note: Void effects on eddy current distortion in two-phase liquid metal
}

\author{
M. Kumar, ${ }^{1,2} \mathrm{Ph}$. Tordjeman, ${ }^{1, \text { a) }}$ W. Bergez, ${ }^{1}$ and M. Cavaro ${ }^{2}$ \\ ${ }^{1}$ Université de Toulouse, Institut de Mécanique des Fluides de Toulouse, Allée du Professeur Camille Soula, \\ 31400 Toulouse, France \\ ${ }^{2}$ CEA, Cadarache, DEN/DTN/STCP/LIET, Building 202, 13108 St Paul Lez Durance, France
}

\begin{abstract}
A model based on the first order perturbation expansion of magnetic flux in a two-phase liquid metal flow has been developed for low magnetic Reynolds number $\mathrm{Re}_{\mathrm{m}}$. This model takes into account the distortion of the induced eddy currents due to the presence of void in the conducting medium. Specific experiments with an eddy current flow meter have been realized for two periodic void distributions. The results have shown, in agreement with the model, that the effects of velocity and void on the emf modulation are decoupled. The magnitude of the void fraction and the void spatial frequency can be determined from the spectral density of the demodulated emf.
\end{abstract}

Characterization of void fraction in two-phase liquid metal is a challenging issue in many applications. The measurement of void fraction in liquid metal is a notoriously difficult problem because these materials are opaque, aggressive, often very hot, and inaccessible. For example, the presence of bubbles in the primary loop of a sodium cooled fast nuclear reactor modifies the neutronic and heat transfer properties of flow, which is a cause of concern from the safety point of view. ${ }^{1}$ In metallurgy, bubbles and non metallic impurities decrease the efficiency of heating by induction and change dramatically the mechanical properties of the manufactured products. ${ }^{2}$ On the other hand, bubbles manifest also in natural magnetohydrodynamics flows, as observed in geophysics earth's outer core or in interstellar medium. ${ }^{3}$ In recent years, we have seen a renewed interest in techniques based on Faraday induction ${ }^{4,5}$ and Lorentz force ${ }^{6}$ for flow measurements. ${ }^{7}$ From a theoretical point of view, the distortion of the induced eddy currents by the presence of void is an open problem. ${ }^{8}$ In this paper, we propose a perturbative theory that allows us to develop a methodology to characterize the void fraction using a standard sensor. ${ }^{9,10}$

We consider a moving two phase liquid metal with a void fraction $\alpha$ and a characteristic velocity $U$. The medium is assumed to flow through a primary coil $P$, which is excited by an AC current $I=I_{0} \cos \omega t$. The perturbation of the induced magnetic flux due to the Faraday and Lorentz force effects is measured by two secondary coils $\left(S_{1}\right.$ and $\left.S_{2}\right)$ placed coaxially on either sides of the primary coil. This corresponds to a standard configuration in flow measurement with the advantage to minimize external noise and, in our case, to amplify the distortion due to void fraction. The model consists to calculate the void fraction contribution to the emf difference between the two secondary coils $\Delta V$. In this problem, the magnetic Reynolds number $\mathrm{Re}_{\mathrm{m}}$ is defined by the penetration depth of the magnetic field in the medium as the characteristic length $\left(\delta=\sqrt{\frac{2}{\sigma \mu_{0} \omega}}\right): \operatorname{Re}_{\mathrm{m}}=\sigma \mu_{0} U \delta$, where $\sigma$ is the electrical conductivity of the medium and $\mu_{0}$ is

\footnotetext{
a)Electronic mail: philippe.tordjeman @imft.fr
}

the magnetic permeability of vacuum. At low $\operatorname{Re}_{\mathrm{m}}$ (diffusion dominates advection in magnetic flux transport equation), the amplitude of the net flux crossing coils $S_{1}$ and $S_{2}$ is given at first order by

$$
\phi=\int \vec{B} \cdot \vec{d} s=\phi_{0}+\operatorname{Re}_{\mathrm{m}} \phi_{u}+\phi_{\alpha}(t)
$$

where $\phi_{0}$ is the average flux in the absence of motion due to Faraday effects, $\phi_{u}$ is the average flux due to Lorentz force effects, and $\phi_{\alpha}$ is the perturbation of the total flux due to the dispersed phase. This last term has its origin in the perturbation of the eddy currents in presence of the non-conducting dispersed phase and is related to the induction effects to first order. In case of periodic distribution of the dispersed phase, this term can be expanded in Fourier series,

$$
\phi_{\alpha}(t)=\psi_{\alpha} \cos \left(\omega_{\alpha} t+\theta\right)+\cdots,
$$

where the amplitude $\psi_{\alpha}$ depends on the volume fraction $\alpha$ and $\omega_{\alpha}$ characterizes the spatial distribution $\left(\omega_{\alpha}=U k_{\alpha}\right.$, where $k_{\alpha}$ is the spatial frequency of dispersed phase). In this analysis, $\omega_{\alpha} \ll \omega$. One notes that $\phi_{0}=0$ for ideally well balanced $S_{1}$ and $S_{2}$. Magnetic field in the medium results from the coupling between the Maxwell-Faraday and the MaxwellAmpere equations, considering that the eddy currents in the conducting media in motion are $\vec{J}=\sigma(\vec{E}+\vec{U} \times \vec{B})$. Consequently, the magnetic field in the medium has two contributions, one in phase and one in quadrature with $I$ $\left(\vec{B}(t)=\vec{B}_{\|} \cos \omega t+\vec{B}_{\perp} \sin \omega t\right)$, which leads to a total flux also with both components. $\Delta V$ is given by differentiating with time the flux between $S_{1}$ and $S_{2}: \Delta V^{2}=\Delta V_{\|}^{2}+\Delta V_{\perp}^{2}$. Considering the first terms in $O\left(\operatorname{Re}_{\mathrm{m}}\right)\left(\operatorname{Re}_{\mathrm{m}} \ll 1\right)$,

$$
\begin{aligned}
\Delta V^{2} \approx & \omega^{2}\left\{\left\langle\phi_{0} \mid \phi_{0}\right\rangle+\frac{\left\langle\psi_{\alpha} \mid \psi_{\alpha}\right\rangle}{2}+2\left\langle\phi_{0} \mid \psi_{\alpha}\right\rangle \cos \left(\omega_{\alpha} t+\theta\right)\right. \\
& +\frac{\left\langle\psi_{\alpha} \mid \psi_{\alpha}\right\rangle}{2} \cos \left(2 \omega_{\alpha} t+2 \theta\right) \\
& \left.+2 \operatorname{Re}_{\mathrm{m}}\left[\left\langle\phi_{0} \mid \phi_{u}\right\rangle+\left\langle\phi_{u} \mid \psi_{\alpha}\right\rangle \cos \left(\omega_{\alpha} t+\theta\right)\right]\right\} .
\end{aligned}
$$

In this formula, $\phi_{i}$ is defined as a vector, $\phi_{i}=\left[\phi_{i, \|}, \phi_{i, \perp}\right]$. $\left\langle\phi_{i} \mid \phi_{j}\right\rangle$ represents the scalar product between the two vectors 


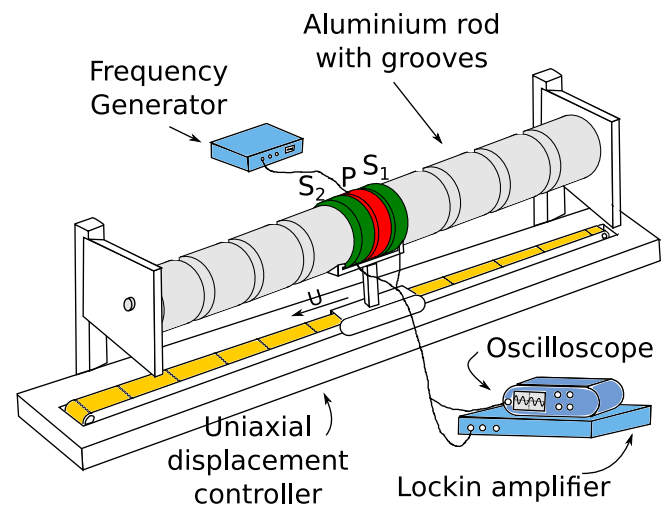

FIG. 1. Design of the experimental set up. $P, S_{1}$, and $S_{2}$ are, respectively, the primary and the two secondary coils.

$i$ and $j$. In RHS of Equation (3), the four first terms correspond to the dominant components in Fourier expansion of $\Delta V^{2}$ and the last terms are the perturbations induced by motion. In Equation (3), only the amplitudes of $\phi_{0}$ and $\psi_{\alpha}$ appear at zero order, and $\left\langle\phi_{u} \mid \phi_{u}\right\rangle$ being a second order term in $\operatorname{Re}_{\mathrm{m}}$ does not appear. The terms $\left\langle\phi_{0} \mid \psi_{\alpha}\right\rangle,\left\langle\phi_{0} \mid \phi_{u}\right\rangle$, and $\left\langle\phi_{u} \mid \psi_{\alpha}\right\rangle$ represent the coupling effects of Faraday induction, Lorentz force, and dispersed phase. The Fourier analysis of the $\Delta V^{2}$ signal allows to determine these components and particularly the term $\left\langle\psi_{\alpha} \mid \psi_{\alpha}\right\rangle$, which is expected to characterize the influence of the dispersed phase.

We have developed specific experiments to validate this theoretical approach. A moving Eddy Current Flow Meter $(\mathrm{ECFM})^{11}$ assembly was designed (Fig. 1). It consists of three coils $\left(P, S_{1}\right.$, and $\left.S_{2}\right)$ of diameter $40 \mathrm{~mm}$ each and length $30 \mathrm{~mm}$ for $P$ and $20 \mathrm{~mm}$ for $S_{1}$ and $S_{2}$, with copper winding of 70 turns for $P$ and 50 for $S_{1}$ and $S_{2}$. ECFM is fixed on a uniaxial displacement controller, which can move at velocity, $U=10^{-3}-1 \mathrm{~m} / \mathrm{s}$. The conducting fluid is modeled by an aluminium rod along which the ECFM moves (see Fig. 1). Three aluminium rods (of diameter $38.5 \mathrm{~mm}$ ) were used in the experiments: a plain rod which represents single phase liquid metal $(\alpha=0 \%)$ and two rods with grooves $(\alpha=0.3 \%$ and $\alpha=6.9 \%$ ), which represent two-phase liquids. The grooves were machined at the rod surface with a period of $18.00 \mathrm{~mm}$ and $16.85 \mathrm{~mm}$, a depth $0.38 \mathrm{~mm}$ and $4.23 \mathrm{~mm}$, and a width $1.4 \mathrm{~mm}$ and $3.00 \mathrm{~mm}$, respectively. The advantage of this system is that the geometric void fraction $\alpha$ is exactly known and there are no problems of liquid metal circulation. The experiment was realized at room temperature for which the electrical conductivity of the aluminium rod is $\sigma=3.8 \times 10^{7} \mathrm{~S} / \mathrm{m}$. We use a lockin amplifier (HF2LI-MF, Zurich Instruments) to excite the primary coil at $3142 \mathrm{rad} / \mathrm{s}$ $(\delta \approx 3.7 \mathrm{~mm})$ and $6283 \mathrm{rad} / \mathrm{s}(\delta \approx 2.6 \mathrm{~mm})$. The amplitude of current intensity for all the experiments is in the range of 200-400 mA. All the experimental results are given for a normalized intensity amplitude of $1 \mathrm{~A}$. Moreover, we have checked that the current remains constant within $0.1 \%$ during an experiment. The voltage induced in $S_{1}$ and $S_{2}$ is measured by the lockin amplifier. In the experiments, the phase of the primary current is taken as reference. $\Delta V$ is demodulated with the lockin amplifier in order to determine the components in phase and in quadrature. For each measurement, the ECFM

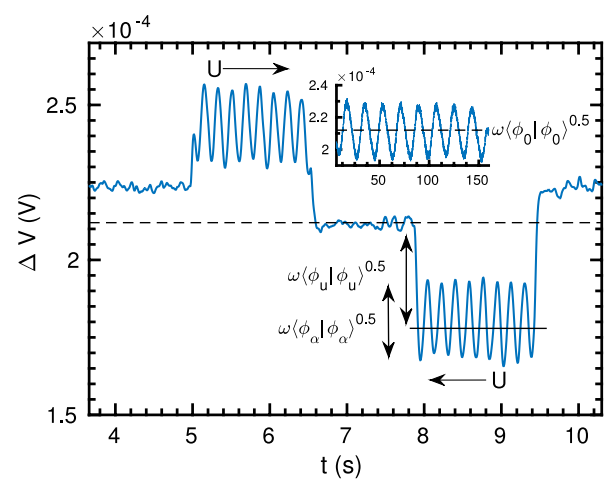

FIG. 2. Typical demodulated voltage difference of the two secondary coils, $\Delta V$ measured vs time for $\alpha=0.3 \%$ at $\omega=3142 \mathrm{rad} / \mathrm{s}$ and $U=0.1 \mathrm{~m} / \mathrm{s}$ (inlay: $U=0.001 \mathrm{~m} / \mathrm{s})$.

was translated back and forth several times. Experiments with this system are very reproducible because the system is highly deterministic. Finally, we have verified that the electromagnetic background noise is not significant at these frequencies.

Fig. 2 displays a typical demodulated $\Delta V$ signal. This kind of signal can be obtained for in phase and quadrature components and also for the norm. $\phi_{0}$ is defined as the average value of the signal when there is very slow motion, typically $U=10^{-3} \mathrm{~m} / \mathrm{s}$ (inlay of Fig. 2). In this case, the Lorentz force can be neglected. In motion at constant $U$, the average value of the signal is translated with a mean amplitude proportional to $\operatorname{Re}_{\mathrm{m}} \phi_{u}$. In presence of grooves $(\alpha \neq 0 \%)$, this signal oscillates around this value. The amplitude of this oscillation gives $\psi_{\alpha}$. Fig. 3 shows an example of the FFT spectrum of the difference between $\Delta V$ signal and its temporal average value for $\alpha=6.9 \%$ at $U=0.1 \mathrm{~m} / \mathrm{s}$ and $6283 \mathrm{rad} / \mathrm{s}$. We observe the first three harmonic peaks in $\omega_{\alpha}$. From the FFT spectral density, we obtained $\omega_{\alpha}=0.35 \pm 0.05 \mathrm{rad} / \mathrm{s}$. This value is in agreement with that calculated from the geometry of grooves: $\omega_{\alpha}=0.37 \mathrm{rad} / \mathrm{s}$. For $\alpha=0.3 \%$, we measured $\omega_{\alpha}=0.35 \pm 0.04$ for a theoretical value $\omega_{\alpha}=0.35$. All the spectra obtained from the experiments validate the expansion at first order in $\mathrm{Re}_{\mathrm{m}}$ of the magnetic flux and also the Fourier series of the void fraction perturbation on eddy current equations (1) and (2). The amplitude of the peak at $3 \omega_{\alpha}$ gives the first residue of Equation (3).

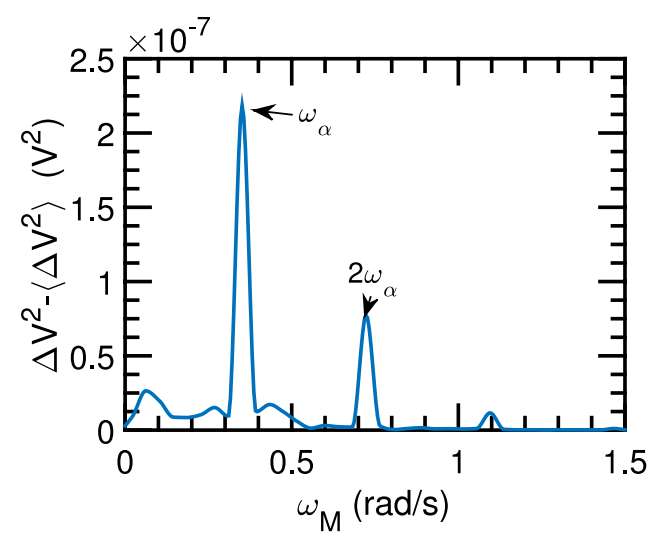

FIG. 3. FFT spectral density of $\left\|\Delta V^{2}\right\|$ vs the modulating pulsation $\omega_{\mathrm{M}}$ for $\alpha=6.9 \%$ and $U=0.001 \mathrm{~m} / \mathrm{s}$ at $6283 \mathrm{rad} / \mathrm{s}$. 

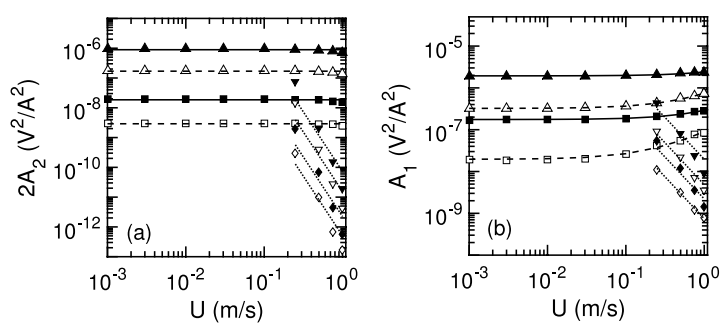

FIG. 4. Amplitude of the second (a) and first (b) peaks of the spectral density of $\|\Delta V\|^{2}$ vs velocity at $3142 \mathrm{rad} / \mathrm{s}$ (unfilled markers) and $6283 \mathrm{rad} / \mathrm{s}$ (filled markers): $\alpha=0.3 \%(\square, \boldsymbol{\square})$ and $(\diamond, \diamond)$ with low pass filter $24 \mathrm{~dB} /$ oct; $\alpha=6.9 \%(\triangle, \mathbf{\Lambda})$ and $(\nabla, \mathbf{\nabla})$ with low pass filter $24 \mathrm{~dB} /$ oct.

According to this equation, the amplitude of the first peak is $A_{1}=\omega^{2}\left[\left\langle\phi_{0} \mid \psi_{\alpha}\right\rangle+2 \operatorname{Re}_{\mathrm{m}}\left\langle\phi_{u} \mid \psi_{\alpha}\right\rangle\right]$ and the amplitude of the second peak is $A_{2}=\omega^{2} \frac{\left\langle\psi_{\alpha} \mid \psi_{\alpha}\right\rangle}{2}$. We have checked that the value of $\sqrt{2 A_{2}} / \omega$ is equal to the norm of $\psi_{\alpha}$, which has been obtained from the direct FFT of $\phi_{\alpha}$ signal.

Fig. 4(a) compares $2 A_{2}$ vs velocity for $\alpha=0.3 \%$ and $\alpha=6.9 \%$ and for both frequencies, 3142 and $6283 \mathrm{rad} / \mathrm{s}$. These experiments showed that $A_{2}$ is independent of the velocity whatever the frequency and the void fraction. On the other hand, the magnitude of $A_{2}$ depends on the void fraction and the frequency. The experimental data show that $\sqrt{A_{2}} / \omega$ is a constant (within $12 \%$ error). Furthermore the experiments point out that the $\alpha$ dependence of $A_{2}$ is given by $\psi_{\alpha} \sim \alpha^{n}, n<1$. In the limit of the accuracy, we found $n \approx 0.6$ for both frequencies. Comparison with the plain rod shows that $A_{2}$ is very sensitive to the presence of void fraction even at low value of $\alpha(\alpha=0.3 \%)$.

For groove depth smaller than $\delta$, the effective void fraction is $\alpha_{\delta} \sim \alpha / \delta$, and if $\alpha$ is small enough, $\psi_{\alpha} \sim \alpha(n=1)$. In this case, we found from the Maxwell's equations that $\psi_{\alpha} \sim \omega^{0}$, in agreement with experiments. The experimental value $n \approx 0.6$ corresponds to the case where groove depth is larger than $\delta$ and $\alpha_{\delta}>\alpha$.

The amplitude of $A_{1}$ vs velocity is shown in Fig. 4(b) for $\alpha=0.3 \%$ and $\alpha=6.9 \%$ at both angular frequencies $3142 \mathrm{rad} / \mathrm{s}$ and $6283 \mathrm{rad} / \mathrm{s}$. We recall that $A_{1}$ characterizes the coupling effects of the void fraction on Faraday induction and on the Lorentz force. In our first order expansion model, $A_{1}$ is linear with $\psi_{\alpha}$. Fig. 4(b) points out that the value of $A_{1}$ increases with $\alpha$, following the same power law relation $\psi_{\alpha} \sim \alpha^{0.6}$. For the two frequencies, we observe that $A_{1}$ is linear with velocity, coming from the coupling term $2 \omega^{2} \operatorname{Re}_{\mathrm{m}}\left\langle\phi_{u} \mid \psi_{\alpha}\right\rangle$.
Due to the order of magnitude of $\left\langle\phi_{0} \mid \psi_{\alpha}\right\rangle$ compared to $2 \operatorname{Re}_{\mathrm{m}}\left\langle\phi_{u} \mid \psi_{\alpha}\right\rangle$, the velocity effects on $A_{1}$ is more visible at low values of $A_{1}$ in log-log representation. Finally, the exact concordance between $A_{1}$ and $A_{2}$ validates the perturbation model given by Equation (3).

To further investigate the consistency of $\psi_{\alpha}$ dynamics on $A_{1}$ and $A_{2}$, we used a low pass filter at a cutoff frequency $v_{c}=6.8 \mathrm{~Hz}$. This filter is characterized by an attenuation of $24 \mathrm{~dB} /$ oct in signal intensity. Since $\omega_{\alpha} \sim U$, the corresponding critical velocity is around $U_{c} \sim 10^{-1} \mathrm{~m} / \mathrm{s}$. After $U_{c}$, the $A_{1}$ and $A_{2}$ values are supposed to decrease in $1 / U^{2}$ and $1 / U^{4}$, respectively. Fig. 4 show that the experimental data verify the expected dynamics.

In this work, we have calculated the effects of the void fraction of a two-phase liquid metal flow on the induced magnetic flux. We have shown that for a periodic void distribution, the amplitude of the emf difference is modulated at a frequency $\omega_{\alpha}$ characteristic of the distribution and with an amplitude which is a function of $\alpha$. We have shown the interest to analyze the FFT signal of demodulated emf difference in order to determine the void fraction. This is possible because the effects of velocity and void are decoupled. The experiments with a standard ECFM validates the perturbation approach used in the model. For more realistic non-periodic void distributions, this approach can still be used to analyze the void effects on emf signal as long as $\omega_{\alpha}$ can be measured.

The authors acknowledge Hervé Ayroles and Grégory Ehses at IMFT Toulouse for their technical assistance. This work has been financially supported by CEA, Cadarache.

${ }^{1}$ A. Judd, J. Phys. D: Appl. Phys. 2, 261 (1969).

${ }^{2}$ M. Lalpoor, D. Eskin, and L. Katgerman, Mater. Sci. Eng.: A 497, 186 (2008).

${ }^{3}$ N. Gehrels and W. Chen, Nature 361(6414), 706-707 (1993).

${ }^{4}$ P. Sharma, S. S. Kumar, B. Nashine, R. Veerasamy, B. Krishnakumar, P. Kalyanasundaram, and G. Vaidyanathan, Ann. Nucl. Energy 37, 332 (2010).

${ }^{5}$ F. Stefani, T. Gundrum, and G. Gerbeth, Phys. Rev. E 70, 056306 (2004).

${ }^{6}$ A. Thess, E. Votyakov, and Y. Kolesnikov, Phys. Rev. Lett. 96, 164501 (2006).

${ }^{7}$ J. Priede, D. Buchenau, and G. Gerbeth, Meas. Sci. Technol. 22(5), 055402 (2011).

${ }^{8} \mathrm{~J}$. C. Maxwell, A Treatise on Electricity and Magnetism (Clarendon Press, 1881), Vol. 1.

${ }^{9}$ C. C. Feng, W. Deeds, and C. V. Dodd, J. Appl. Phys. 46, 2935 (1975).

${ }^{10}$ K. Nakamoto, S. Tamura, K. Ishii, H. Kuwahara, N. Ohyamaand, and T. Muramatsu, Nucl. Eng. Des. 82, 393 (1984).

${ }^{11}$ H. Lehde and W. T. Lang, U.S. patent 2,435,043 (27 January 1948). 